detection and typing of dengue viruses. J Clin Virol. 2009;45:61-6. https://doi.org/10.1016/j.jcv.2009.02.010

6. Baronti C, Piorkowski G, Leparc-Goffart I, de Lamballerie X, Dubot-Pérès A. Rapid next-generation sequencing of dengue, EV-A71 and RSV-A viruses. J Virol Methods. 2015;226:7-14. https://doi.org/10.1016/j.jviromet.2015.09.004

7. Ayolabi CI, Olusola BA, Ibemgbo SA, Okonkwo GO. Detection of dengue viruses among febrile patients in Lagos, Nigeria and phylogenetics of circulating dengue serotypes in Africa. Infect Genet Evol. 2019;75:103947. https://doi.org/ 10.1016/j.meegid.2019.103947

8. Wang Y, Wang X, Liu X, Ren R, Zhou L, Li C, et al. Epidemiology of imported infectious diseases, China, 2005-2016. Emerg Infect Dis. 2018;25:33-41. https:/ / doi.org/ 10.3201/eid2501.180178

Address for correspondence: Antoine Nougairède, Faculté de médecine, 27 Bd Jean Moulin, 13005 Marseille, France; email: antoine.nougairede@univ-amu.fr

\section{Outbreak of Human Metapneumovirus Infection in Zoo, Slovenia}

Tina Uršič, Nika Lalek, Pavel Kvapil, Marjan Kastelic, Vasilij Cociancich, Irena G. Košnik, Miroslav Petrovec

Author affiliations: University of Ljubljana, Ljubljana, Slovenia

(T. Uršič, V. Cociancich, M. Petrovec); Golnik University Clinic of Pulmonary and Allergic Diseases, Golnik, Slovenia (N. Lalek); Ljubljana Zoo, Ljubljana (P. Kvapil, M. Kastelic); National Institute of Public Health, National Laboratory of Health, Environment, and Food, Maribor, Slovenia (I.G. Košnik)

DOI: https://doi.org/10.3201/eid2608.200125

We report a case of human metapneumovirus infection that spread from humans to chimpanzees and back to humans. Bronchopneumonia developed in 4 of 6 members of a chimpanzee family, and 2 subsequently died. The chimpanzees' keeper also became ill. Sequencing showed $100 \%$ identity between virus sequences from chimpanzees and the keeper.

A pes are the closest nonhuman primate relative of humans and are therefore susceptible to many human pathogens. Chimpanzees have been kept at the Ljubljana Zoo in Slovenia since 1974. The zoo had never experienced a severe or fatal case of viral respiratory infection among the chimpanzee family, which consisted of 6 members $(10,11,13,15,25$, and 38 years of age). We report an outbreak of human metapneumovirus (hMPV) infection in chimpanzees and a zookeeper at this zoo.

This study was performed in accordance with the Helsinki Declaration. Written consent was obtained from the human patient and archived.

On June 19, 2013, four of the youngest chimpanzees at the zoo started showing signs of a cold (snorting, sneezing, and coughing). Veterinarians suspected a viral infection, but because of the possibility of secondary bacterial infection, the chimpanzees were given amoxicillin and clavulanic acid. The next day, clinical signs of pneumonia (apathy, dyspnea, and loss of appetite) appeared. The youngest chimpanzee died of respiratory failure on June 22; necropsy showed acute bronchopneumonia.

We tested animals for influenza A and B viruses, respiratory syncytial virus, hMPV, human coronaviruses (NL63, OC43, HKU1, and 229E), human bocavirus 1 , human rhinoviruses, adenoviruses, and enteroviruses by using real-time reverse transcription PCR. Only hMPV was detected in nasal and throat swab specimens and lung tissue (1). The same day, the health of the other chimpanzees deteriorated.

The second-youngest chimpanzee that had signs of acute respiratory distress was sedated, ventilated, and given Ringer solution, bronchodilators, and intravenous antimicrobial drugs. However, it died on June 24 because of bronchopneumonia and pleural effusion, which was confirmed by necropsy.

In nasal and throat swab specimens and lung tissue, only hMPV and Klebsiella pneumoniae were detected. Histopathologic examination of hematoxylin and eosin-stained lung tissue samples of both chimpanzees that died showed severe bronchointerstitial pneumonia, including necrosis and sloughing of bronchial and bronchiolar epithelium; alveolar spaces filled with an exudate composed of foamy macrophages, neutrophils, and fibrin; and multifocal intraalveolar hemorrhage. In the second chimpanzee, we observed multifocal manifestations of bacilli-like bacteria. For the remaining 2 ill chimpanzees, clinical signs gradually disappeared in 6 days.

The keeper of the chimpanzees was a 31-year-old man, a nonsmoker who had a history of persistent allergic rhinoconjunctivitis and childhood asthma. He reported signs of a cold on June 21. His health deteriorated over the next 2 days; he had fever, sore 


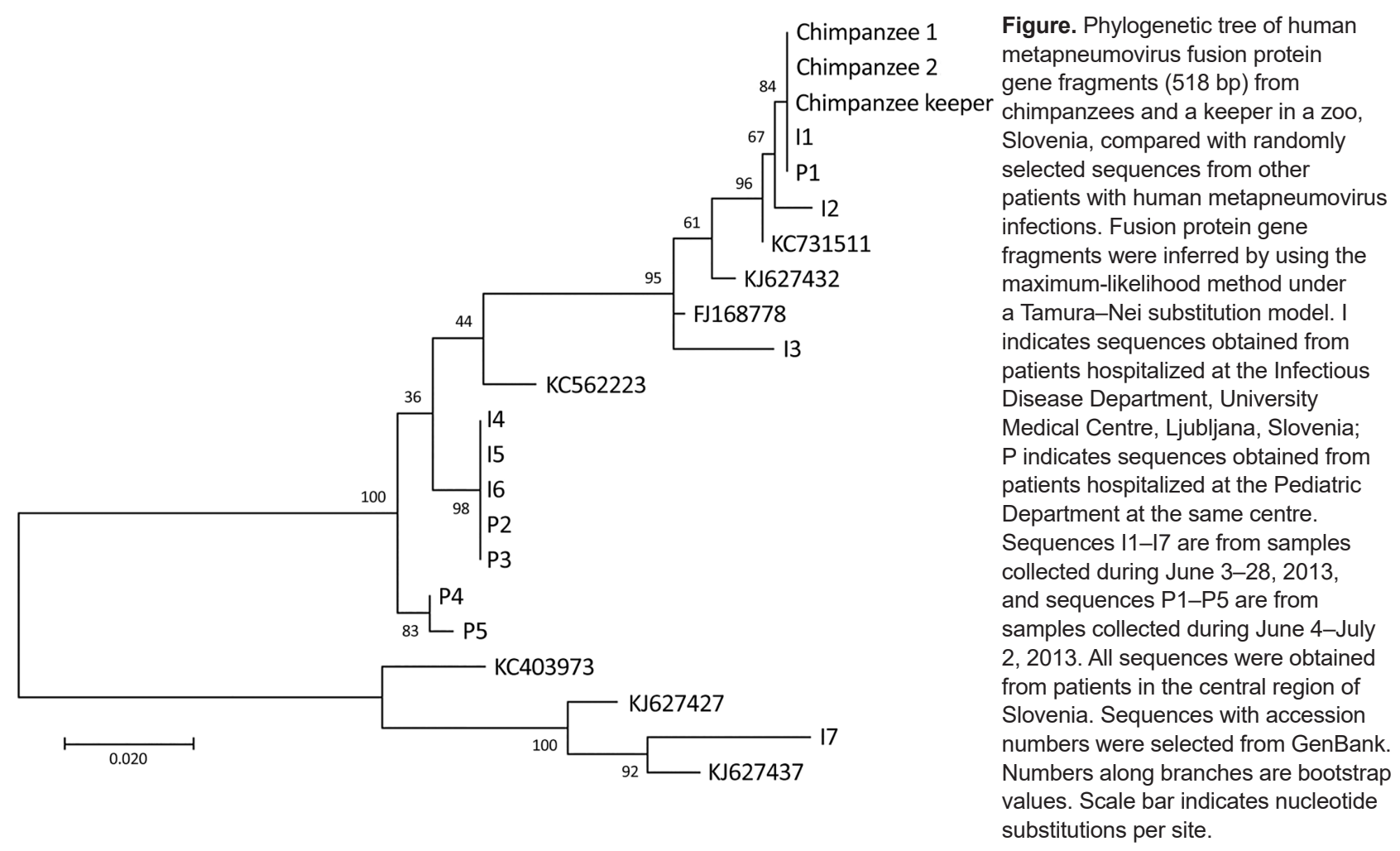

throat, chills, muscle and joint pain, headache, and a dry cough. He visited the emergency department of Golnik University Clinic of Pulmonology and Allergic Diseases on June 23.

A nasopharyngeal swab specimen showed only $\mathrm{hMPV}$ by PCR. A throat swab specimen was negative for pathogenic bacteria. On July 1, the patient was hospitalized because of chest tightness and wheezing. Examination detected inspiratory and expiratory wheezing with crepitations. High-resolution computed tomography showed localized mucus plugs in the bronchi for right posterior basal segments with segmental air trapping and no radiologic signs of asthma or bronchiolitis. He was given inhaled methylprednisolone and high-dose salbutamol and was discharged on July 4.

To confirm that the respiratory infection had spread from chimpanzees to their keeper, we performed realtime reverse transcription PCR and sequenced PCR products of part of the hMPV fusion protein gene (2). At the time of the hMPV outbreak among the chimpanzees, hMPV was circulating in Slovenia. Among 39 samples available for June 3-July 2 from the population in central Slovenia, we sequenced 12 samples with the lowest cycle threshold values to facilitate insight into the molecular epidemiology of circulating hMPV variants.

Sequencing analyses showed that virus sequences from the chimpanzees and keeper had 100\% identity (Figure), as did 2 other sequences from 2 patients hospitalized during the virus outbreak at the zoo. These 2 sequences showed $99 \%$ identity with a previously reported sequence (GenBank accession no. KC731511). Alignment of all 15 sequences and phylogenetic analyses showed 7 unique sequences (GenBank accession nos. MN978917-23) among the 12 selected samples.

These cases are noteworthy because of how the infection spread. We assume that the chimpanzees acquired the virus from an infected child in a school or preschool group of children visiting the zoo as an end-of-year trip. The infection could have been spread by airborne route. Another possibility is that the chimpanzees acquired the virus from contaminated food of visitors; because of a narrow space $(1.3 \mathrm{~m})$ between visitors and the inner fence enclosing the chimpanzee cage, children could have thrown candies and other food onto the cage floor, and the chimpanzees might have eaten them.

Although the keeper had close contact with an ill chimpanzee and was probably infected by aerosols or body fluids from the chimpanzees, we cannot rule out the possibility that the keeper could have been infected by an infected adult human. We assume high infectivity pressure on the keeper, who fell ill only 2 days after the first chimpanzee started showing signs of respiratory infection. The keeper did not have children of his own and did not have close contact with children for at least 2 weeks before the incident. 
This study was partially supported by the Slovenian Research Agency (Research Program P3-0083).

\section{About the Author \\ Dr. Uršič is a research scientist at the Institute of Microbiology and Immunology, University of Ljubljana, Ljubljana, Slovenia. Her primary research interests are severe respiratory viral infections in children and the elderly, and molecular epidemiology studies.}

\section{References}

1. Maertzdorf J, Wang CK, Brown JB, Quinto JD, Chu M, de Graaf M, et al. Real-time reverse transcriptase PCR assay for detection of human metapneumoviruses from all known genetic lineages. J Clin Microbiol. 2004;42:981-6. https://doi.org/10.1128/JCM.42.3.981-986.2004

2. Reiche J, Jacobsen S, Neubauer K, Hafemann S, Nitsche A, Milde J, et al. Human metapneumovirus: insights from a ten-year molecular and epidemiological analysis in Germany. PLoS One. 2014;9:e88342. https:/ / doi.org/ 10.1371/journal.pone.0088342

Address for correspondence: Tina Uršič, Institute of Microbiology and Immunology, Faculty of Medicine, University of Ljubljana, Zaloška 4, 1000 Ljubljana, Slovenia; email: tina.ursic@mf.uni-lj.si

\section{mcr-Positive Escherichia coli ST131-H22 from Poultry in Brazil}

\author{
Andre Becker S. Saidenberg, Marc Stegger, \\ Lance Bradley Price, Thor Bech Johannesen, \\ Maliha Aziz, Marcos P.V. Cunha, Andrea M. Moreno, \\ Terezinha Knöbl
}

\footnotetext{
Author affiliations: University of São Paulo, São Paulo, Brazil

(A.B.S. Saidenberg, M.P.V. Cunha, A.M. Moreno, T. Knöbl);

George Washington University, Washington, DC, USA

(M. Stegger, L.B. Price, M. Aziz); Statens Serum Institut,

Copenhagen, Denmark (M. Stegger, T.B. Johannesen)

DOI: https://doi.org/10.3201/eid2608.191724
}

Escherichia coli sequence type (ST) 131 is of concern because it can acquire antimicrobial resistance and cause extraintestinal infections. E. coli ST131-H22 sublineage appears capable of being transmitted to humans through poultry. We report on multidrug-resistant ST131H22 poultry isolates in Brazil closely related to international human and poultry isolates.

The pandemic, extraintestinal, pathogenic Esch1 erichia coli multilocus sequence type (MLST) 131 lineage has emerged extensively, gaining notoriety for its extensively multidrug-resistant ST131-H30 sublineage (1). Whereas ST131-H30 appears to be transmitted primarily from person to person, the $\mathrm{H} 22$ sublineage may be transmitted zoonotically through poultry and cause urinary tract infections and urosepsis $(2,3)$. We report isolating ST131-H22 strains that are multidrug resistant (MDR), meaning that they are resistant to $\geq 3$ classes of antimicrobials (4), carrying mobile colistinresistance ( $\mathrm{mcr}$ ) determinants from poultry in Brazil, the largest poultry-exporting country in the world.

We collected $64 \mathrm{E}$. coli strains from poultry with colibacillosis cases from 2 different farms in the same geographic region of Brazil and screened them by PCR for the ST131 clonal group (5). PCR detected 6 ST131 isolates (2 from the first farm, 4 from the second), which we whole-genome sequenced (BioProject no. PRJNA398035). We determined phenotypic antimicrobial susceptibility with disk diffusion testing, except for isolates carrying the mor gene, which we tested using broth microdilution (6).

We trimmed the reads and used QUAST (http:/ / quast.sourceforge.net) to evaluate the quality of assemblies (contig lengths and expected genome sizes). We assembled DNA sequences with SPAdes (http:/ / cab.spbu.ru/software/spades), then determined the serotype, phylogroup, MLST, fim H protein type, virulence gene profile, plasmid replicons, and markers of antimicrobial resistance for each isolate in silico using the ABRicate virulence factors database (https:/ / github.com/tseemann/abricate) and ResFinder/ PlasmidFinder tools from CGE (https://cge.cbs.dtu. $\mathrm{dk} /$ services). Genes were identified with a minimum of $\geq 95 \%$ of identity and coverage.

We identified all isolates as O25:H4-ST131-H22, all belonging to phylogroup B2. We generated a maximum-likelihood phylogeny tree on the basis of coregenome single-nucleotide polymorphisms, including the 6 isolates from Brazil and 140ST131-H22 sequences from EnteroBase (http://enterobase.warwick.ac.uk) and a previous study (2), using the Northern Arizona SNP Pipeline (https://tgennorth.github.io/NASP/) aligned against E. coli JJ1886 ST131-H30 (GenBank 